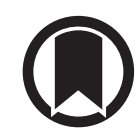

CrossMark

\section{DNA methylation modules in airway smooth muscle are associated with asthma severity}

\author{
To the Editor:
}

Asthma is a chronic airway inflammatory disorder characterised by airway hyperresponsiveness, inflammation and remodelling, including airway smooth muscle cell (ASMC) hyperplasia and subepithelial airway fibrosis $[1,2]$. ASMCs from severe asthmatics are hyperproliferative, release more pro-inflammatory cytokines and are corticosteroid-insensitive compared with those from healthy individuals and non-severe asthma patients $[3,4]$. Genetic and epigenetic processes such as miRNA expression and DNA methylation have been implicated in asthma pathogenesis [5]. Indeed, DNA methylation is altered in asthmatic blood cells [5] and may be a biomarker of atopy [6].

We hypothesised that genome-wide analysis of DNA methylation associated with altered mRNA and miRNA expression would reveal insights into pathways driving severe asthma particularly in ASMCs whose function is abnormal in disease. We analysed the interconnections and functional relevance of differences in DNA methylation status and the expression of mRNAs and miRNAs in ASMCs cultured from bronchial biopsies obtained from five healthy subjects, five non-severe asthma patients (NSA) and five severe asthma patients (SA), at baseline and following stimulation with $2.5 \%$ fetal calf serum (FCS) and transforming growth factor (TGF)- $\beta\left(1 \mathrm{ng} \cdot \mathrm{mL}^{-1}\right)[3,4]$. This stimulus is known to induce ASMC proliferation and enhance the release of inflammatory mediators in a severity dependent manner $[3,4]$. SA and NSA were defined as previously described $[1,2]$. Healthy subjects had no previous history of asthma and provocative concentration causing a $20 \%$ fall in forced expiratory volume in $1 \mathrm{~s}>16 \mathrm{mg} \cdot \mathrm{mL}^{-1}$. Full patient demographics are shown in table 1. The study was approved by the Ethics Committee of the Royal Brompton and Harefield Hospital NHS Trust and all subjects gave written informed consent.

DNA methylation analysis using the Illumina $450 \mathrm{~K}$ array (Infinium HumanMethylation450 K v1.2, San Diego, CA, USA) identified 12383 differentially methylated positions (DMPs) (defined by $>20 \%$ mean methylation difference), which clustered by clinical designation (figure 1a). Analysis of DMPs between groups revealed 15 hub sites (figure $1 \mathrm{~b}$ ) that, apart from TRPV1, are associated with novel asthma genes linked to important processes/pathways implicated in asthma pathophysiology including inflammatory, metabolic and proliferative pathways in ASMCs from SA.

Pathway analysis using g:Profiler [7] indicated that the genes associated with DMPs in healthy subjects versus NSA at baseline were involved in the regulation of cell proliferation and apoptosis (Hippo signalling pathway) and axon guidance, while differences between SA and controls were associated with ASMC contraction (calcium signalling pathway), proliferation (cancer-related pathways) and the endocytosis pathway (figure 1c). Stimulation of healthy subjects ASMCs activated pathways similar to those seen in the NSA and SA cells (hippo pathway and endocytosis). This suggests that ASMCs from NSA and SA retain an epigenetic profile linked to cell proliferation and asthma despite being in culture for several passages. Stimulated NSA ASMCs increased DMPs linked with repression of innate immune responses (EpsteinBarr virus infection), while in SA cells, the Notch signalling pathway was most significantly activated. This data highlights the importance of altered innate immunity and proliferative signalling by Notch in severe asthma ASMCs.

We used a different approach to examine the importance of severity associated DMPs, namely weighted gene co-expression analysis (WGCNA) [8] and Bumphunter analysis [9]. We identified five out of 19

@ERSpublications

Abnormal DNA methylation patterns distinguish airway smooth muscle cell function in asthma and asthma severity http://ow.ly/cTrK30iCwVK

Cite this article as: Perry M, Lavender P, Kuo C-hS, et al. DNA methylation modules in airway smooth muscle are associated with asthma severity. Eur Respir J 2018; 0: 1701068 [https://doi.org/10.1183/ 13993003.01068-2017]. 
TABLE 1 Patient demographics

\begin{tabular}{lccc} 
& $\begin{array}{c}\text { Healthy } \\
\text { subjects }\end{array}$ & $\begin{array}{c}\text { Non-severe asthma } \\
\text { patients }\end{array}$ & $\begin{array}{c}\text { Severe asthma } \\
\text { patients }\end{array}$ \\
\hline Subjects & 5 & 5 & 5 \\
Age years & $36.4 \pm 12.72$ & $42.1 \pm 16.06$ & $40.9 \pm 11$ \\
Males:females & $3: 2$ & $2: 3$ & $0: 5$ \\
Duration of asthma years & $\mathrm{N} / \mathrm{A}$ & $21.2 \pm 15.32$ & $25.56 \pm 13.24$ \\
Inhaled corticosteroid dose $\boldsymbol{\mu g}$ BDP equivalent & 0 & $480 \pm 582.71$ & $1688.89 \pm 176.38$ \\
Atopy & 0 & 3 & 5 \\
Receiving oral corticosteroids & 0 & 0 & 5 \\
FEV $\mathbf{L}$ & $4.02 \pm 0.48$ & $2.7 \pm 0.82$ & $2.7 \pm 0.77$ \\
FEV 1 \% predicted & $104.23 \pm 7.28$ & $84.48 \pm 18.34$ & $81.42 \pm 14.82$ \\
FEV1/FVC \% & $78.89 \pm 5.98$ & $73.98 \pm 9.67$ & $69.62 \pm 9.31$ \\
PC20 $\mathbf{m g} \cdot \mathbf{m L}^{-1}$ & $>16$ & $0.75 \pm 9.31$ & Not performed
\end{tabular}

Data are presented as $\mathrm{n}$ or mean \pm SD. BDP: beclomethasone diproprionate; $F E V_{1}$ : forced expiratory volume in $1 \mathrm{~s}$; FVC: forced vital capacity; PC20: provocative concentration causing a 20\% fall in FEV1.

modules as being significantly correlated with disease severity (figure 1d). Filtering of these phenotype-associated CpG sites for significance and connectivity gave 15 phenotype DMPs that were more enriched in genomic $\mathrm{CpG}$ shores or regions immediately flanking $\mathrm{CpG}$ islands and confirmed the importance of the previously identified $D B X 2, A C P 6$ and KCNJ11 as hub DMPs. These genes are associated with altered fatty acid metabolism and proliferation (ACP6), G-protein-coupled receptor signalling in metabolic syndrome (KCNJ11) and neuronal patterning (DBX2).

This analysis also confirmed the previously reported decrease in baseline PDE4D (phosphodiesterase 4D) promoter region methylation in asthma that modulated asthmatic ASMC proliferation [10]. However, this effect was reversed when cells were stimulated with FCS/TGF- $\beta$ highlighting that ASMC DNA methylation status is labile upon cell activation to induce functional consequences. Pathway analysis of phenotype-associated DMPs confirmed the importance of the Hippo signalling, axon guidance and endocytosis pathways, but also delineated novel pathways including endocannabinoid signalling, which is typically present in the brain [11] and in viral induction of tumours [12]. Further investigation of the role of these pathways in SA is required.

DNA methylation not only regulates gene expression, but also that of miRNAs. Altered expression of miRNAs regulates many physiological processes, including inflammation and remodelling, and has been implicated in asthma [3]. We examined whether the DMPs described here may also control miRNA expression particularly as one of the 15 hub DMPs is associated with miR-548a-3 (figure $1 \mathrm{~b}$ ). We found altered expression of numerous miRNAs (measured by reverse transcriptase (RT-)PCR as previously described $[3,13,14]$ ) whose loci were linked with DMPs in asthmatic ASMC at baseline and following stimulation. 113 DMPs associated with miRNA loci were seen comparing healthy subjects and NSA patients; 104 when comparing healthy subjects and SA samples and 120 when comparing NSA and SA ASMCs (figure 1e). The miRNAs associated with the highest and lowest methylated CpGs being MIR137 and MIR372, respectively, in the healthy subjects ASMCs, MIR548Q and MIR575 in the NSA samples, and MIR1265 and MIR1266 in ASMCs from SA. MIR137, MIR372 and MIR575 have been reported to be aberrantly expressed in both ASMCs [13] and asthmatic lung biopsies [14]. However, we show for the first time that $m i R-1265$ and $m i R-1266$ are overexpressed in SA ASMCs.

Stimulation with FCS/TGF- $\beta$ had a greater effect on DMPs in SA cells, with significantly altered DMPs seen at 10 miRNAs in healthy subjects ASMCs, 18 in NSA and 50 in SA ASMCs at false discovery rate (figure 1f). The highest and lowest methylated positions, respectively, in healthy subjects ASMCs were associated with MIR218-1 and MIR548F5, with MIR613 and MIR125B1 in NSA samples, and with MIR663 and MIR320D1 in the ASMCs from SA (figure 1f). Using RT-PCR, we confirmed the expected change in expression of the mature miRNAs correlating with the respective increase or decrease in methylation status. The most highly-expressed miRNA was miR-320d-1 in the SA ASMC, confirming our previous reports in both ASMCs [13] and asthmatic lung biopsies [14] (figure 1f). In summary, our data suggests that regulation of proliferative/apoptotic pathways is abnormal in ASMC from SA patients and that this may be under the control of dysregulated metabolic processes, particularly those relating to fatty acid metabolism.

Overall, there was $\sim 80 \%$ overlap of the DNA methylation profiles reported here and with gene expression profiles from healthy subjects and NSA and SA primary ASMCs as previously published $[5,13,14]$. This 

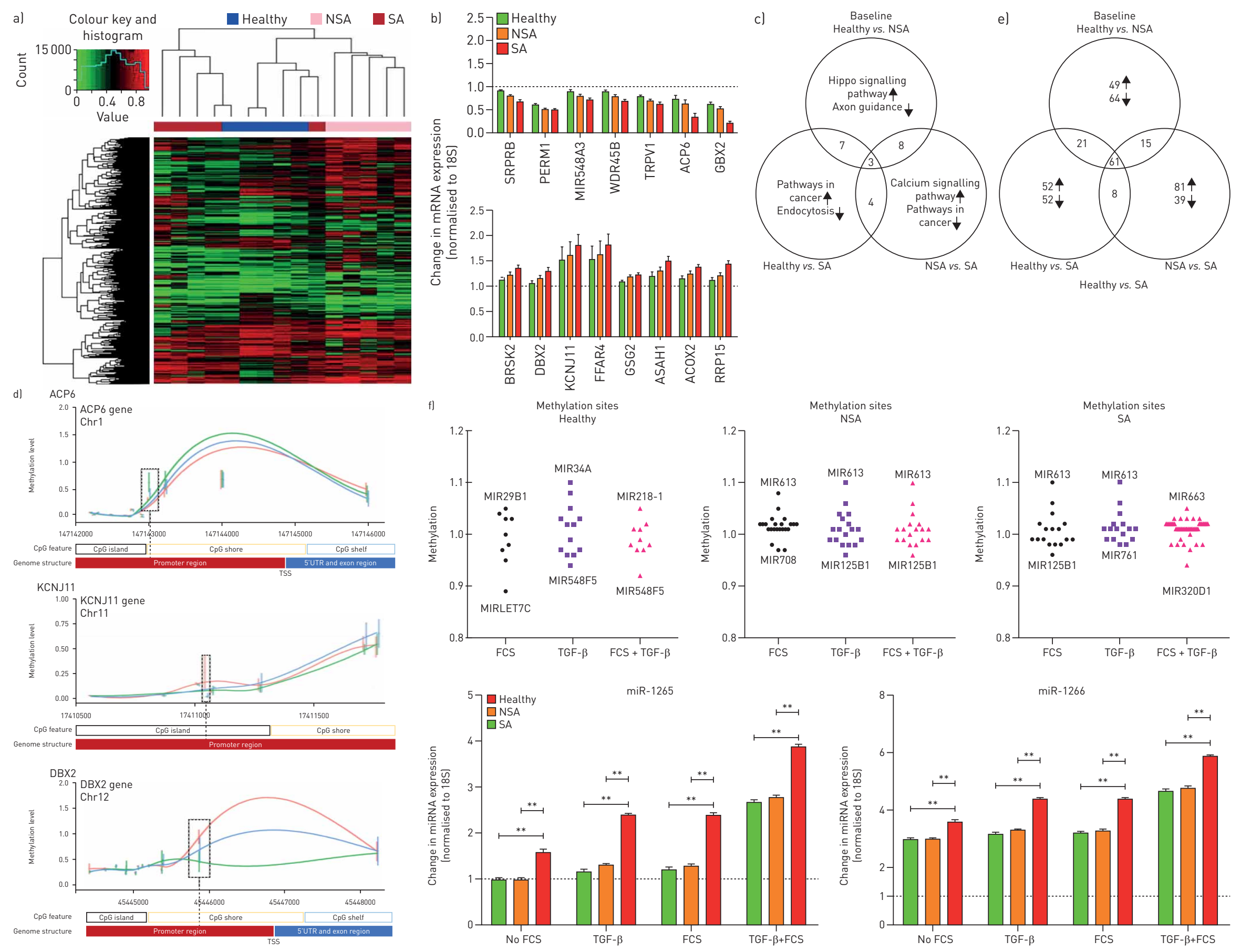
FIGURE 1 DNA methylation sites and miRNA regulation in severe asthma airway smooth muscle cells. a) Heatmap showing global methylation pattern of airway smooth muscle. Unsupervised hierarchical clustering of 12383 differentially methylated DNA sites. Red represents high methylation and green represents low methylation. b) Change in RNA expression of the 15 "hub" genes, as measured by reverse transcriptase PCR. c) Pathway analysis of genes associated with differentially methylated positions (DMPs). d) The genomic location of three the hub DMPs suggested by both weighted gene co-expression analysis (WGCNA) and bumphunter analysis, are shown. The KCNJ11 DMP sits in both the CpG island and promoter regions, whereas ACP6 and DBX2 sit in both CpG shore and promoter regions. e) Venn diagram showing inter-group comparison of differential methylation sites associated with miRNAs at baseline. f) Graphical representation of the numbers of differentially expressed miRNAs sites in healthy, non-severe asthma (NSA) and severe asthma (SA) airway smooth muscle following stimulation with fetal calf serum (FCS) $(2.5 \%)$ and transforming growth factor (TGF)- $\beta\left(1 \mathrm{ng} \cdot \mathrm{mL}^{-1}\right)$, both at the methylation level and miRNA level.

not only confirms our focused analysis at the mRNA level but also suggests that factors such as diet and lifestyle, known to affect DNA methylation status [15], are unlikely to cause a major bias here. Larger focused studies will be required to address these issues.

We confirm that distinct patterns of DNA methylation are associated with asthma per se and also with disease severity. These methylation changes, in turn, are associated with changes in gene expression and miRNA expression which may influence ASMC function. Indeed, we describe a new mechanism underlying ASMC dysfunction in SA and provide a rationale for delineating the potential therapeutic action of demethylating agents (i.e. adjuvant treatment). We also highlight the potential role of novel pathways such as endocannabinoid signalling in mediating ASMC function in SA. Therefore, this study not only extends our understanding of the multi-layered or integrated nature of the regulatory mechanisms that control the SA ASMC phenotype, but it should stimulate more work on the functional regulation of ASMC function in SA with the potential for newer more effective therapies aimed at not only relieving the increased airway tone but also the hyperproliferative and proinflammatory ASMC of severe asthma.

Mark M. Perry ${ }^{1}$, Paul Lavender ${ }^{2}$, Chih-hsi Scott Kuo ${ }^{3}$, Francesca Galea ${ }^{4}$, Charalambos Michaeloudes ${ }^{5}$, James M. Flanagan ${ }^{5}$, Kian Fan Chung ${ }^{5}$ and Ian M. Adcock ${ }^{5}$

${ }^{1}$ School of Pharmacy and Biomedical Sciences, University of Portsmouth, Portsmouth, UK. ${ }^{2}$ MRC and Asthma UK Centre in Allergic Mechanisms of Asthma, Kings College London, London, UK. ${ }^{3}$ Discovery Science Group, Dept of Computing, Imperial College London, London, UK. ${ }^{4}$ Epigenetics Unit, Dept of Surgery and Cancer, Imperial College London, London, UK. ${ }^{5}$ Airways Disease, National Heart and Lung Institute, Imperial College, London and Royal Brompton NIHR Biomedical Research Unit, London, UK.

Correspondence: Mark M. Perry, School of Pharmacy and Biomedical Sciences, University of Portsmouth, St. Michael's Building, White Swan Road, Portsmouth, PO1 2DT, UK. E-mail: mark.perry@port.ac.uk

Received: Sept 212016 | Accepted after revision: Feb 082018

Authors' contributions: M.M. Perry planned the experiments, cultured the ASM cells, analysed the data and prepared the manuscript; P. Lavender ran the DNA methylation arrays; C.H.S. Kuo analysed the data; F. Galea, C. Michaeloudes and J.M. Flanagan were responsible for the pyrosequencing; K.F. Chung recruited the patients and performed the bronchial biopsies; I.M. Adcock funded the study. M.M. Perry, K.F. Chung and I.M. Adcock designed the study. Consent for publication: All authors have read the manuscript and consent to it being published. Availability of data and material: All data and material are included in the manuscript.

Conflict of interest: None declared.

Support statement: This work was supported by grants from Asthma UK (08/041) and The Wellcome Trust (085935) (K.F. Chung). This project was supported by the NIHR Respiratory Disease Biomedical Research Unit at the Royal Brompton and Harefield NHS Foundation Trust and Imperial College London. The views expressed in this publication are those of the authors(s) and not necessarily those of the NHS, The National Institute for Health Research or the Department of Health. K.F. Chung is a Senior Investigator of NIHR, UK. I.M. Adcock and K.F. Chung are supported by the EU-Innovative Medicines Initiative Joint Undertaking project U-BIOPRED (115010). M.M. Perry, I.M. Adcock and K.F. Chung are members of Interuniversity Attraction Poles Program-Belgian State-Belgian Science Policy- project P7/ 30. Funding information for this article has been deposited with the Crossref Funder Registry.

\section{References}

1 Chung KF, Wenzel SE, Brozek JL, et al. International ERS/ATS guidelines on definition, evaluation and treatment of severe asthma. Eur Respir J 2014; 43: 343-373.

2 Reddel HK, Bateman ED, Becker A, et al. A summary of the new GINA strategy: a roadmap to asthma control. Eur Respir J 2015; 46: 622-639.

3 Perry MM, Baker JE, Gibeon DS, et al. Airway smooth muscle hyperproliferation is regulated by microRNA-221 in severe asthma. Am J Respir Cell Mol Biol 2013; 50: 7-17.

4 Chang PJ, Bhavsar PK, Michaeloudes C, et al. Corticosteroid insensitivity of chemokine expression in airway smooth muscle of patients with severe asthma. J Allergy Clin Immunol 2012; 130: 877-885.

5 Potaczek DP, Harb H, Michel S, et al. Epigenetics and allergy: from basic mechanisms to clinical applications. Epigenomics 2017; 9: 539-571. 
6 Liang L, Willis-Owen SA, Laprise C, et al. An epigenome-wide association study of total serum immunoglobulin E concentration. Nature 2015; 520: 670-674.

7 Reimand J, Arak T, Vilo J. g:Profiler-a web server for functional interpretation of gene lists (2011 update). Nucleic Acids 2011; 39: W307-W315.

8 Langfelder P, Horvath S. WGCNA: an R package for weighted correlation network analysis. BMC Bioinformatics 2008; 9: 559.

9 Jaffe AE, Murakami $\mathrm{P}$, Lee $\mathrm{H}$, et al. Bump hunting to identify differentially methylated regions in epigenetic epidemiology studies. Int J Epidemiol 2012; 41: 200-209.

10 Lin AH, Shang Y, Mitzner W, et al. Aberrant DNA methylation of phosphodiesterase 4D: effect on airway smooth muscle cell phenotypes. Am J Respir Cell Mol Biol 2016; 54: 241-249.

11 Di Marzo V, Stella N, Zimmer A. Endocannabinoid signalling and the deteriorating brain. Nat Rev Neurosci 2015; 16: 30-42.

12 Pierangeli A, Antonelli G, Gentile G. Immunodeficiency-associated viral oncogenesis. ClinMicrobiolInfect 2015; 21: 975-983.

13 Perry M, Tsitsiou E, Austin PJ, et al. Role of non-coding RNAs in maintaining primary airway smooth muscle cells. Respir Res 2014; 15: 58.

14 Williams AE, Larner-Svensson H, Perry MM, et al. MicroRNA expression profiling in mild asthmatic human airways and effect of corticosteroid therapy. PLoS One 2009; 4: e5889.

15 Lim U, Song MA. Dietary and lifestyle factors of DNA methylation. Methods Mol Biol 2012; 863: 359-376.

Copyright (CERS 2018

This ERJ Open article is open access and distributed under the terms of the Creative Commons Attribution Licence 4.0. 